\title{
Prediction of a Blast Furnace Burden Distribution Variable
}

\author{
Mats NIKUS and Henrik SAXÉN \\ Heat Engineering Laboratory, Department of Chemical Engineering, Åbo Akademi University, Biskopsgatan 8, FIN-20500 \\ Åbo, Finland. E-mail: henrik.saxen@abo.fi
}

(Received on January 8, 1996; accepted in final form on April 12, 1996)

\begin{abstract}
A model for prediction of a variable that characterizes the burden distribution in the blast furnace is presented. The neural network model makes use of short-term temperature measurements from an above-burden probe as well as information about the charging program, and predicts a normalized temperature change, which can be considered to reflect the burden distribution. The basic structure of both an on-line and an off-line model is presented. By applying the models on process data from a Finnish blast furnace it is demonstrated that accurate predictions are obtained of the temperature changes caused by the dumps for the entire charging cycle.
\end{abstract}

KEY WORDS: burden distribution estimation; blast furnace; above-burden probe; neural networks.

\section{Introduction}

The fuel economy of the blast furnace process depends to a large extent on the gas distribution, which strongly influences both the thermal and the chemical phenomena in the lumpy zone of the furnace. The gas distribution, which also affects the pressure loss as well as productivity and smoothness of operation, is controlled mainly by manipulating the distribution of the burden. The burden distribution is, in itself, of considerable importance, since it affects the shape of the cohesive zone, which is known to play a decisive role in the operation of the blast furnace. This work focuses on methods for estimation of burden distribution in furnaces with bells.

A number of efforts have been made to clarify the burden distribution in furnaces with bell-type charging equipment. One way of tackling the problem is to carry out small-scale experiments, with pilot models of the furnace top constructed according to similarity criteria based on dimensional analysis, ${ }^{1,2)}$ or full-scale experiments. ${ }^{3)}$ Usually, the experiments have been carried out without paying attention to the effect of the gas flow, but this can lead to serious misinterpretation of the burden distribution. ${ }^{2,4)}$ Furthermore new experiments are required if the conditions (e.g. geometry, material properties) change in the real process.

Another approach is to tackle the problem by theoretical modeling of the trajectories of the burden particles as they fall from the large bell, ${ }^{3)}$ further considering the segregation of the material on the burden surface ${ }^{2,5,6)}$ Such work has led to sophisticated models, by which changes in geometry and material properties can be studied. However, values have to be found for a large number of parameters (particle diameters or size distribution, shape and friction factors, porosities, etc.), and pilot experiments should, in general, be undertaken to verify the theoretical models.

A third approach is to utilize direct or indirect measurements from operating furnaces to gain insight into the factors that affect the burden distribution. By analyzing measurements from above-burden or inburden probes, which record temperatures and/or gas composition at different radial positions, information about the over-all gas and burden distribution can be obtained. ${ }^{7,8)}$ Various more sophisticated measurement methods have also been developed. ${ }^{9}{ }^{9}$ The burden surface has been detected physically by profile meters, ${ }^{7}$ by radar, ${ }^{10,11)}$ microwaves or laser, ${ }^{9)}$ as well as IR and gamma radiation. ${ }^{12)}$ Also, the distribution of gas (and burden) can be indirectly studied by thermo-cameras, which measure the burden surface temperture, ${ }^{13.14)}$ and the layer thickness can be measured by magnetometer ${ }^{5)}$ or by microwave techniques. ${ }^{15)}$

The method for on-line estimation of burden distribution presented in this paper is based on a novel approach, ${ }^{16,17)}$ which does not require installation of expensive measurement devices or sensors: When material is dumped on the burden surface from the large bell, a change in the gas temperature measured by an above-burden probe will be observed, because of heat transfer between the ascending gas and the charged burden. The magnitude of the short-term change mainly depends on the local layer thickness of the charged burden, so it is suggested that the temperature measurements can be used to estimate the burden distribution. In the literature, only Cheng et al. ${ }^{18,19)}$ seem to have pointed out that records of such cooling of the gas could be used for similar purposes, while most investigators consider these temperature changes merely as disturbances.

This paper outlines the approach and presents a 
preliminary analysis of how to predict the temperature changes, caused by charging, in the central part of the furnace. Using process data from a Finnish blast furnace, equipped with an above-burden probe with $K=11$ temperature measurements, $T_{i}, i=1, \ldots, K$, it is demonstrated that the temperature drops can be predicted accurately.

\section{The Approach}

\subsection{General Outline}

When a dump of burden falls from the large bell and eventually distributes on the burden surface, changes in the gas temperatures measured by the above-burden probe can usually be observed. Typically, the gas profile collapses because of the arising heat flow from the gas to the charged burden layer, but recovers in a few minutes when the layer has been heated. The changes in the gas temperature due to charging are next analyzed by means of a simplified model. An energy balance for the latest charged layer (Fig. 1) can be written

$$
\dot{m}_{\mathrm{g}} c_{\mathrm{g}}\left[T_{\mathrm{g}, \text { in }}-T_{\mathrm{g}}(t)\right]=m_{\mathrm{s}} c_{\mathrm{s}} \frac{d T_{\mathrm{s}}}{d t}=\dot{Q},
$$

where the gas entering the layer is assumed to have a constant temperature, $T_{\mathrm{g}, \text { in }}$, and the burden is characterized by a uniform temperature, $T_{\mathrm{s}}$. If the heat flow between the gas and the burden, $\dot{Q}$, is expressed as

$$
\dot{Q}=h A\left[T_{\mathrm{g}}(t)-T_{\mathrm{s}}(t)\right],
$$

where $A$ is the heat transfer area and $h$ is the heat transfer coefficient, the two equations can be combined to yield an expression for the outlet temperature of the gas

$$
T_{\mathrm{g}}(t)=\frac{h A T_{\mathrm{s}}(t)+\dot{m}_{\mathrm{g}} c_{\mathrm{g}} T_{\mathrm{g}, \text { in }}}{h A+\dot{m}_{\mathrm{g}} c_{\mathrm{g}}}=\frac{h A T_{\mathrm{s}}(t)+\dot{C}_{\mathrm{g}} T_{\mathrm{g}, \text { in }}}{h A+\dot{C}_{\mathrm{g}}}
$$

where $\dot{C}_{\mathrm{g}}$ is the heat capacity flow of gas. This equaion can also be written as

$$
\frac{T_{\mathrm{g}}(t)-T_{\mathrm{s}}(t)}{T_{\mathrm{g}, \text { in }}-T_{\mathrm{s}}(t)}=\frac{\dot{C}_{\mathrm{g}}}{h A+\dot{C}_{\mathrm{g}}} .
$$

By inserting $t=0$ into Eq. (4)-as we are especially interested in the instantaneous drop in the gas temperature-it can be noted that the heat capacity of the solid phase does not affect the immediate cooling of the gas.

However by studying 1-min mean values of the probe temperatures, which were available at the blast furnace in question, the maximum temperature drop was found to occur on an average two minutes after each dump.

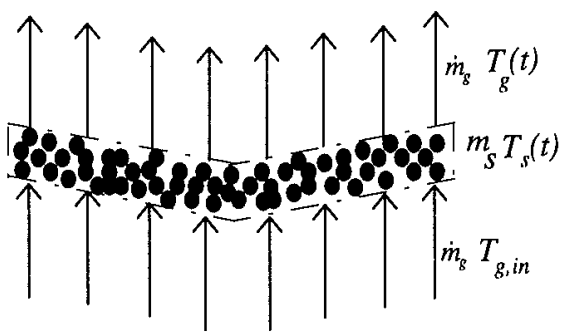

Fig. 1. The balance volume and the notation used.
The slower response can be due to measurement dynamics. This was supported by observations from a test period, where the temperature measurements from the probe were logged every fifth second, showing maximum changes about $80 \mathrm{sec}$ after the dumps (see the Appendix). In order to study the dynamic response of the simplified model, Eq. (3) can be introduced into the differential Eq. (1) to yield

$$
\tau \frac{d T_{\mathrm{s}}}{d t}=T_{\mathrm{g}, \text { in }}-T_{\mathrm{s}}(t)
$$

with

$$
\tau=\frac{C_{\mathrm{s}}}{\dot{C}_{\mathrm{g}}} \cdot \frac{\left(\dot{C}_{\mathrm{g}}+h A\right)}{h A} .
$$

Solving Eq. (5) gives the burden temperature

$$
T_{\mathrm{s}}(t)=T_{\mathrm{g}, \text { in }}-\left[T_{\mathrm{g}, \text { in }}-T_{\mathrm{s}}(0)\right] e^{-t / \tau} .
$$

Finally, the gas temperature is obtained by inserting this result into Eq. (3). An example of simulated temperatures is given in the Appendix.

Based on the above findings, it is now proposed that the burden distribution can be characterized in terms of an auxiliary (normalized) variable, $\beta_{i}$, defined by

$$
\beta_{i}=\frac{T_{\mathrm{g}, i}\left(t_{d}\right)-T_{\mathrm{g}, i}\left(t_{\mathrm{d}}+2 \mathrm{~min}\right)}{T_{\mathrm{g}, i}\left(t_{\mathrm{d}}\right)-T_{\mathrm{s}}} ; \quad i=1, \ldots, K
$$

where $t_{\mathrm{d}}$ is the time of the dump. The equation expresses the temperature change of the gas at the $i$ th probe position after a dump, normalized against the change that would be obtained if the gas temperature, $T_{\mathrm{g}, i}$ changed to that of the charged burden, $T_{\mathrm{s}}$. A motivation for the index is that we can write the transfer area for the heat flow between the gas and the charged layer (from Eqs. (1) and (2)) as

$$
A=\frac{\dot{C}_{\mathrm{g}}}{h} \cdot \frac{T_{\mathrm{g}, \text { in }}-T_{\mathrm{g}}(t)}{T_{\mathrm{g}}(t)-T_{\mathrm{s}}(t)},
$$

where the second term on the right hand side can be approximated with $\beta$. Since $A$ is proportional to the thickness of the charged layer, it follows that the radial distribution of $\beta$ should reflect the distribution of the charged material.

The paper demonstrates that the evolution of $\beta$ for the dumps in the charging program can be expressed as a function of other variables, such as material fraction, temperatures, etc. This indicates that the radial distribution of burden during the charging sequence is deterministic enough to be predicted.

\subsection{Estimation of the Initial Temperature of Burden}

In order to carry out the analysis proposed above, estimates of the initial temperature of the charged material are needed. Since there is no coke plant at the studied iron works, the Koverhar blast furnace in Lappohja, Finland, ${ }^{20)}$ the coke temperature was assumed to be constant. For the sinter, however, large fluctuations in temperature were expected, because this burden comes from the nearby sinter plant (after passing through screening and material bins). It was therefore 
decided that the sinter temperature be measured in a weighing hopper before the burden enters the conveyor belt to the blast furnace top. Because this measurement was slow, the temperature had rarely time to settle before the hopper was emptied. Thus, the sinter temperature had to be estimated from the recorded incomplete responses, $T_{\mathrm{h}}$, depicted by solid lines in Fig. 2 for a short time period.

The dynamics of the measurement in the hopper were found to correspond quite well to a first order system. For the sake of simplicity, possible overlap of responses was neglected treating every step response separately, i.e.,

$$
T_{\mathrm{h}}(t)=T_{\mathrm{h}}\left(t_{0}\right)+\Delta T\left(1-e^{-\left(t-t_{0}\right) / \tau_{\mathrm{h}}}\right),
$$

where $\Delta T=T_{\mathrm{s}}-T_{\mathrm{h}}\left(t_{0}\right)$ is the (theoretical) temperature change, and $t_{0}$ is the time when the first effects of the change are observed. Expressed in time-discrete form, with a time increment of one minute, the hopper temperature during the responses follows $T_{\mathrm{h}}(l)=a T_{\mathrm{h}}(l-1)$ $+b$. Taking the difference between two consecutive time instances, and solving for $a$ yields

$$
a=\frac{T_{\mathrm{h}}(l)-T_{\mathrm{h}}(l-1)}{T_{\mathrm{h}}(l-1)-T_{\mathrm{h}}(l-2)} .
$$

By studying the value of this parameter during the responses (dotted line in Fig. 2) it was found that it, after some initial instability, often settled on a level of $a \approx 0.85$ (dashed horizontal line). By noting that $a=\exp (-\Delta t /$ $\tau_{\mathrm{h}}$ ), we get $\tau_{\mathrm{h}}=-\Delta t / \ln a \approx 6 \mathrm{~min}$. Using this fixed value for all responses, which is a reasonable assumption, an estimate of the temperature step can be determined by least squares for each of the responses from

$$
\Delta \hat{T}=\frac{\sum_{l=1}^{n}\left(1-e^{-l / \tau_{\mathrm{h}}}\right)\left(T_{\mathrm{h}}(l)-T_{\mathrm{h}}(0)\right)}{\sum_{l=1}^{n}\left(T_{\mathrm{h}}(l)-T_{\mathrm{h}}(0)\right)^{2}},
$$

where $n$ is the number of available observations for the step response in question. Finally, the estimated burden temperature is obtained from $T_{\mathrm{s}}=T_{\mathrm{h}}(0)+\Delta \hat{T}$. Some estimated responses are illustrated by dashed-dotted lines in Fig. 2. It was found that the sinter temperatures from different bins could vary as much as $90^{\circ} \mathrm{C}$, the reason being that the routes between the sinter plant and the material bins are not equal for the different fractions (small, medium, and coarse). However, generally, each material bin was found to be characterized by an approximately constant temperature, even though
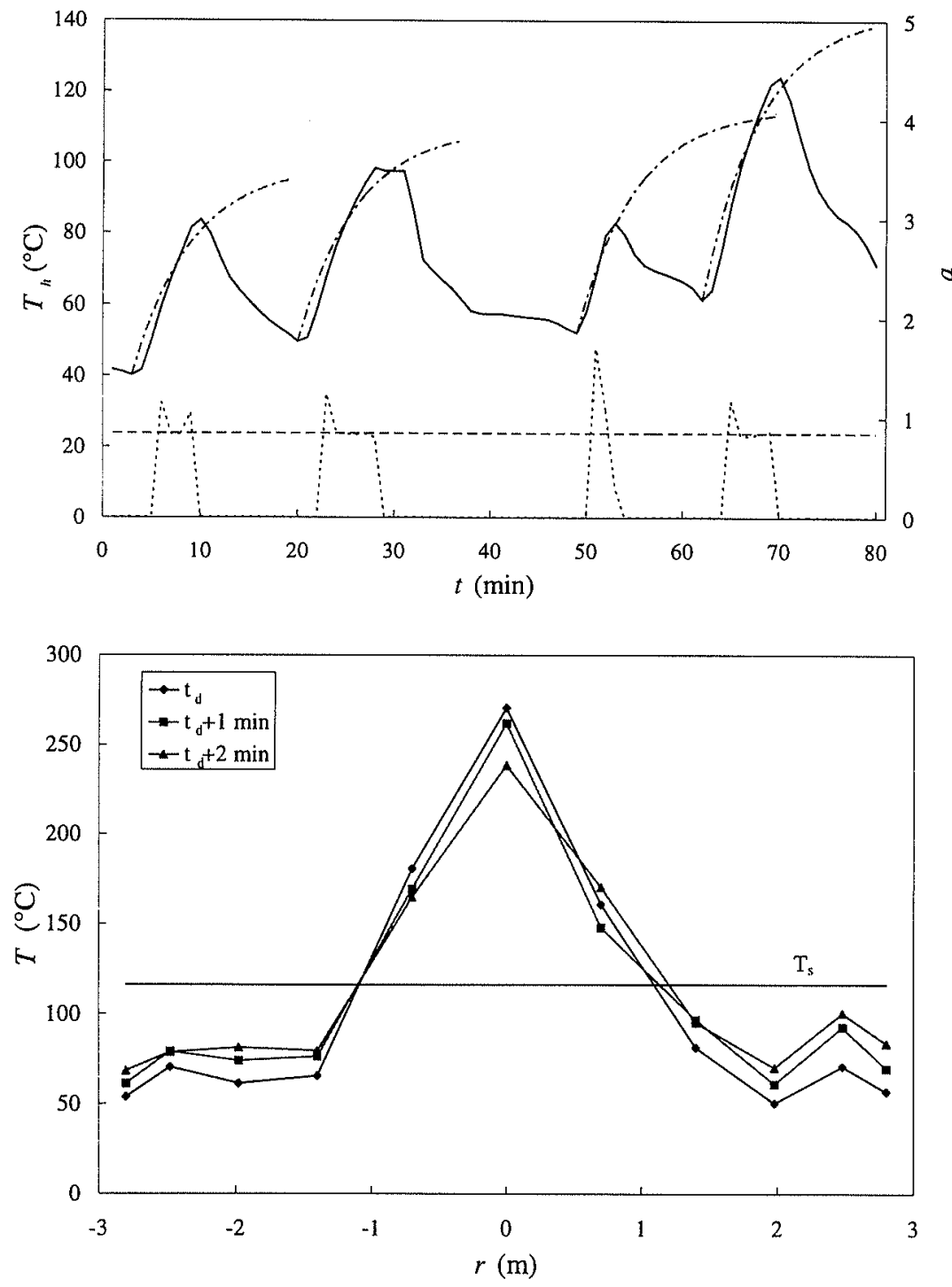

Fig. 2.

Temperature in the weighing hopper (solid line), estimated first order responses (dash-dotted lines), the values of $a$ calucated for the step responses (dotted line) and the constant value used for it in estimating the time constant of the system (dashed line).
Fig. 3.

The evolution of the probe temperatures after a dump of fine sinter at $t_{\mathrm{d}}$. 


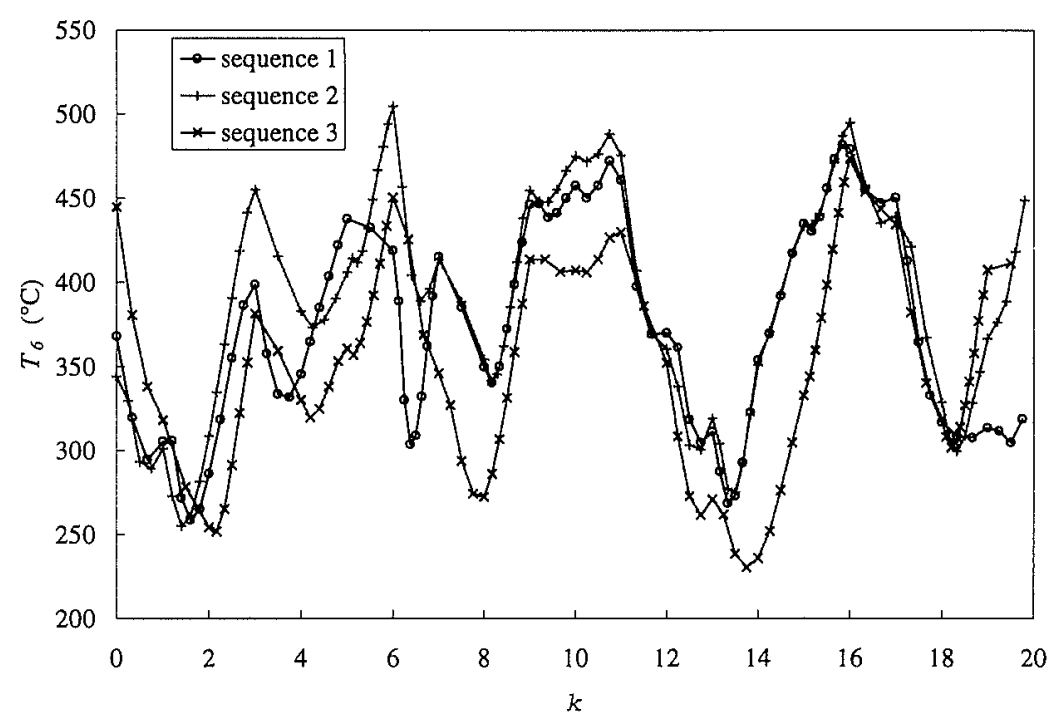

Fig. 4.

The center probe temperature, $T_{6}$, during three consecutive charging sequences. some drift was observed.

Figure 3 shows the changes in probe temperatures after a dump of fine sinter-the warmest burden material at the studied blast furnace. For this specific dump, the sinter temperature was estimated to be $T_{\mathrm{s}}=115^{\circ} \mathrm{C}$. While the gas temperature in the center is cooled by the dump, the temperatures at the periphery clearly rise. This example stresses the importance of considering the burden temperature in the model.

\subsection{Reproducibility of the Burden Distribution}

For the data period studied, an exceptional reproducibility in the probe temperatures was noticed, which coincided with the charging sequence. Figure 4 illustrates the center temperature of the probe, $T_{6}$, for three consecutive charging sequences. (The $K=11$ probe thermocouples are located symmetrically with respect to the center.) In order to emphasize the similarities, the abscissa depicts the dump number in the sequence (which consists of 10 sinter and 10 coke dumps). Between the dumps, the number of (1-minute) observations of the temperatures, depicted by the symbols $O,+$, and $\times$, varies quite considerably, the average being about 4.5 . The striking similarity of the gas temperature suggests that also the burden distribution within the charging sequence is very reproducible. Clearly, for this operation point, there are periods when the burden accumulates at the periphery (gas temperature in the center rises steadily) followed by collapses of the burden into the central region (gas temperature drops dramatically).

\subsection{Modeling}

In the modeling, it was decided to make some assumptions, which were known to be quite restrictive but were introduced in order to keep the model as simple as possible. Possible changes with time in the mass flow rate of gas are neglected. The attention is focused only on the evolution of the center temperature, $T_{6}$, of the probe, because the effect of possible errors in the estimated burden temperature, $T_{\mathrm{s}}$, will not have a dramatic effect on $\beta_{6}$ (since $T_{6} \gg T_{\mathrm{s}}$ ).

Several factors are known to influence the burden distribution, but since the mechanisms are not known well, it was decided to use a black-box approach in the modeling. Because of the observed tendencies to periodicity in $T_{6}$ (Fig. 4), it was decided to include lagged values of the dependent variable, $\beta_{6}(k-1), \beta_{6}(k-2), \ldots$, as inputs, where $k$ denotes the time of the dumps in time-discrete form. Another important variable is the time elapsed between (the previous and the present) dumps, $\Delta t_{\mathrm{d}}(k)=t_{\mathrm{d}}(k)-t_{\mathrm{d}}(k-1)$. A long delay between the dumps may indicate that the previous layer of burden was thick under the stockrods. Further, binary variables indicating the material $(\gamma=1$ for ore, and $\gamma=0$ for coke), and the particle size ( $\delta=1$ for fine, and $\delta=0$ for coarse particles) were included as inputs. For the 3-day data period studied in this work, all sinter dumps entered with movable armor notch 1 , and all coke dumps with notch 2 , so the effect of the armor was here neglected. Obviously, this important variable must be considered in the future.

The sought relationship, $f$, can now be written

$$
\begin{aligned}
& \hat{\beta}_{6}(k)= \\
& \quad f\left(\beta_{6}(k-1), \beta_{6}(k-2), \ldots, \gamma(k), \delta(k), \Delta t_{\mathrm{d}}(k), T_{6}(k)\right)
\end{aligned}
$$

and it was decided to use feedforward neural networks ${ }^{21)}$ to determine the mapping due to their capability to capture nonlinearities. The neural networks studied in this work have an input layer of $N_{\text {inp }}$ units, which simply passes the information forwards to a hidden layer of $N_{\text {hid }}$ sigmoidal units. The prediction, $\hat{\beta}_{6}(k)$, is computed in the single output unit as a weighted sum of the outputs from the hidden units. The networks will in what follows be abbreviated as $\left(N_{\text {inp }}, N_{\text {hid }}, 1\right)$. Linear models are correspondingly denoted by $\left(N_{\text {inp }}, 1\right)$.

The values of $\beta_{6}$ for the time period studied were computed using sinter temperatures estimated by the method described in Sec. 2.2 , while $10^{\circ} \mathrm{C}$ was used as the coke temperature. Two data sets of equal size (390 observations) were formed; one set for training (subscript "tr") and one set for testing (subscript "te") the networks. As a measure of the performance, the root mean square error 

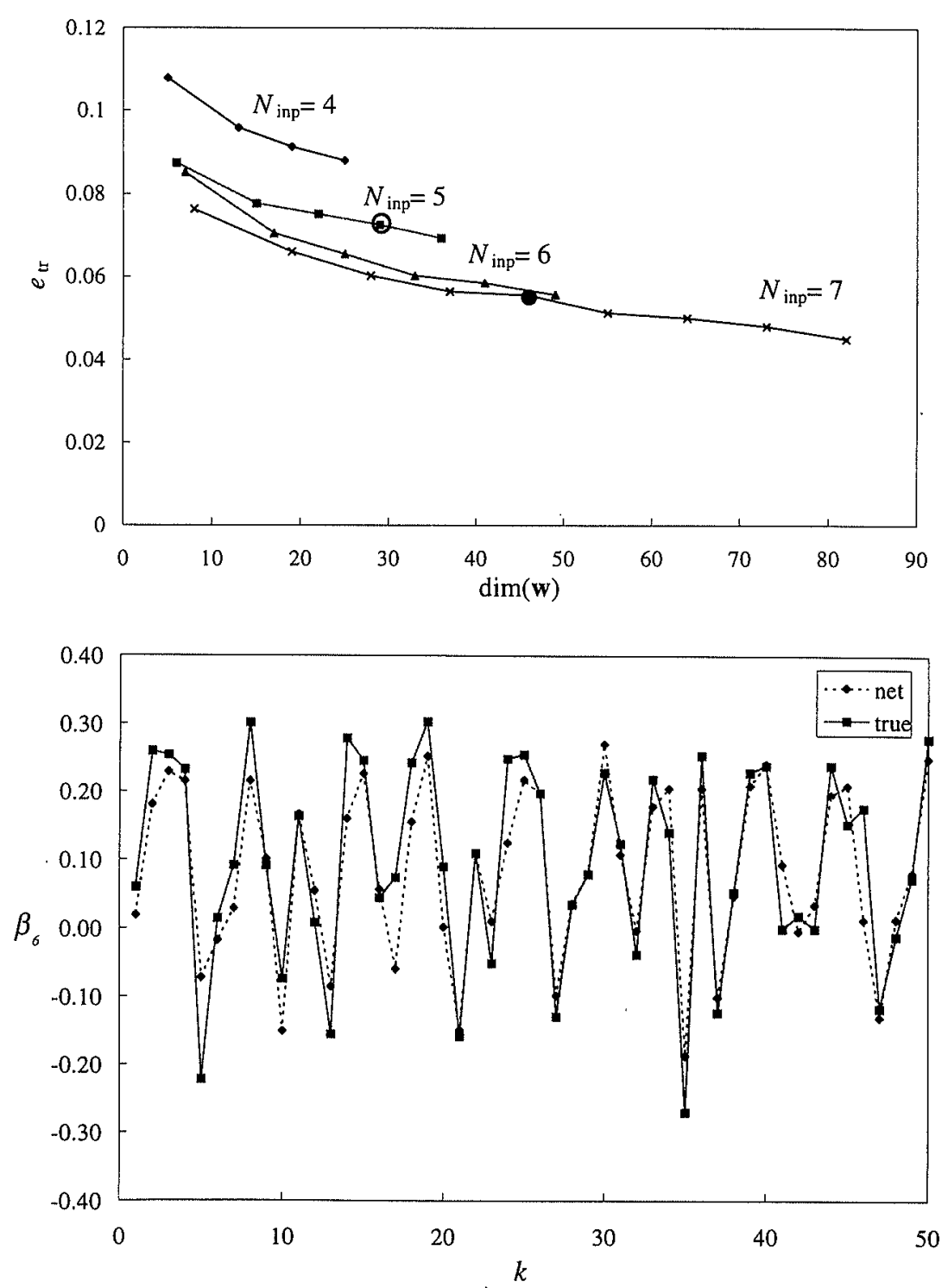

Fig. 5.

The training errors as a function of number of weights for different input dimensions of the feedforward neural networks. The networks indicated by circles are those selected for further analysis.

$$
e_{j}=\sqrt{\frac{\sum_{k=1}^{M_{j}}\left(\hat{\beta}_{6}(k)-\beta_{6}(k)\right)^{2}}{M_{j}}}, j=\mathrm{tr}, \text { te }
$$

was used, where $M_{j}$ is the number of observations in data set $j$. The networks were trained by minimizing the error square sum with respect to the weight vector, w, using the NNDT software. ${ }^{22)}$

\section{Results}

This section presents the results of some alternative models and compares their performance for different input vectors in order to find an "optimal" network size. The models are also made recurrent in order to predict the temperature changes for hypothetical charging programs.

\subsection{Performance of Different Sized Networks}

For different input vectors, linear models were first determined, while the necessity of nonlinearity in the models was investigated by training networks with a hidden layer, with different number of hidden nodes. The choice of model complexity is mainly based on the performance of the models on the test set.

It was found useful to consider three previous values
Table 1. Number of network parameters, and training and test errors for different sized 7-input networks studies.

\begin{tabular}{|c|c|c|c|}
\hline Network & $\operatorname{dim}(\boldsymbol{w})$ & $e_{\mathrm{tr}}$ & $e_{\mathrm{e}}$ \\
\hline$(7,1)$ & 8 & 0.0763 & 0.0767 \\
\hline$(7,2,1)$ & 19 & 0.0659 & 0.0703 \\
\hline$(7,3,1)$ & 28 & 0.0602 & 0.0714 \\
\hline$(7,4,1)$ & 37 & 0.0564 & 0.0640 \\
\hline$(7,5,1)$ & 46 & 0.0555 & 0.0636 \\
\hline$(7,6,1)$ & 55 & 0.0513 & 0.0706 \\
\hline$(7,7,1)$ & 64 & 0.0501 & 0.0691 \\
\hline$(7,8,1)$ & 73 & 0.0481 & 0.0653 \\
\hline$(7,9,1)$ & 82 & 0.0451 & 0.0688 \\
\hline
\end{tabular}

of the dependent variable in the input vector. Generally, very little was gained by using nonlinear models if the input dimension was small, but for $N_{\text {inp }} \geq 5$ a reduction in the test set errors was observed if hidden nodes were included $^{17)}$; this justifies the use of nonlinear models for the task. The larger networks exhibited a slight overfitting effect, i.e., rising test set errors when the number of hidden nodes was increased above a certain limit, but the performance was not especially sensitive to the number of hidden nodes. Figure 5 shows the training errors as a function of the number of weights in networks 
with four different input vectors, the difference being in the last four variables; the input dimension is increased by adding, in turn, $\delta(k), \Delta t_{\mathrm{d}}(k)$, and $T_{6}(k)$ to the common inputs $\beta_{6}(k-1), \beta_{6}(k-2), \beta_{6}(k-3)$, and $\gamma(k)$. A network with the seven inputs $\beta_{6}(k-1), \beta_{6}(k-2)$, $\beta_{6}(k-3), \gamma(k), \delta(k), \Delta t_{\mathrm{d}}(k)$, and $T_{6}(k)$, with five hidden nodes (indicated by the solid circle in Fig. 5), was found to be a good choice, as indicated by the test set errors of Table 1. Figure 6 illustrates the true values of $\beta_{6}$ (solid line) and the network's predictions (dotted line) for 50 instances from the test set. The performance of the model is observed to be good, and it can predict most of the major changes in the value of $\beta_{6}$.

\subsection{Several Step Predictions by Feeding Back Outputs}

An obvious limitation of the applicability of the model presented above is that it is based on recent process measurements. This problem can be partly tackled by using the model recursively by feeding back lagged predicted values, $\hat{\beta}_{6}$, to the input layer. Naturally, this recurrent network requires initial values of the $\beta_{6}$ values, but these can be obtained either by starting from values taken from the data set or by assigning them arbitrary values. If the latter approach is used, the predictions are likely to be poor initially, but will improve as the predictions evolve. A completely autonomous model would be obtained if the last two variables of the input vector, $\Delta t_{\mathrm{d}}$ and $T_{6}$, were excluded, since the only remaining external inputs, $\gamma$ and $\delta$, are uniquely specified by the charging program. Such a model could be very

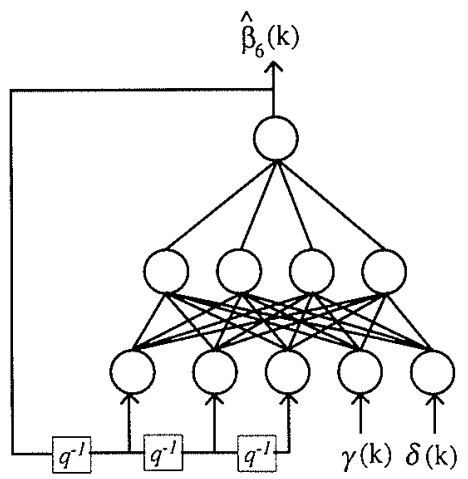

Fig. 7. The $(5,4,1)$ feedback neural network. useful in the design of new charging programs.

Starting from a feedforward net trained on the five inputs, $\beta_{6}(k-1), \beta_{6}(k-2), \beta_{6}(k-3), \gamma(k)$ and $\delta(k)$, and hardwiring this with feedback connections from the output, an autonomous model is obtained. Using a network with four hidden nodes (also indicated in Fig. 5 by the open circle), i.e., a $(5,4,1)$ network, the modification presented in Fig. 7 was obtained. Table 2 lists the input signals for a (20-dump) charging program from the test set, which is used to illustrate the performance of the autonomous network. Note that there are three dumps of fine coke and four dumps of fine sinter in the sequence, some of which clearly "trigger" larger changes in the burden distribution, which result in major drops in the probe temperature, $T_{6}$. Starting from the "true" values of $\beta_{6}$ (taken from the test set), the performance of the feedback $(5,4,1)$ network is shown in Fig. 8. The predictions are not as good as those of the feedforward $(7,5,1)$ network with complete input information, but its over-all prediction capability is entirely satisfactory.

Table 2. Dump number and process variables used as model inputs for the charging program studied, indicating the material type, particle size, time between dumps, and gas temperature.

\begin{tabular}{|c|c|c|c|c|}
\hline$k$ & $\gamma$ & $\delta$ & $\Delta t_{\mathrm{d}} / \min$ & $T_{6} /{ }^{\circ} \mathrm{C}$ \\
\hline 1 & 1 & 1 & 3 & 196 \\
\hline 2 & 0 & 0 & 5 & 191 \\
\hline 3 & 1 & 0 & 5 & 287 \\
\hline 4 & 0 & 0 & 5 & 361 \\
\hline 5 & 1 & 1 & 4 & 378 \\
\hline 6 & 0 & 0 & 5 & 277 \\
\hline 7 & 1 & 0 & 4 & 248 \\
\hline 8 & 0 & 1 & 6 & 309 \\
\hline 9 & 1 & 0 & 3 & 272 \\
\hline 10 & 0 & 0 & 5 & 231 \\
\hline 11 & 1 & 1 & 3 & 285 \\
\hline 12 & 0 & 0 & 5 & 295 \\
\hline 13 & 1 & 0 & 4 & 345 \\
\hline 14 & 0 & 1 & 5 & 377 \\
\hline 15 & 1 & 1 & 4 & 324 \\
\hline 16 & 0 & 0 & 3 & 280 \\
\hline 17 & 1 & 0 & 5 & 281 \\
\hline 18 & 0 & 0 & 8 & 394 \\
\hline 19 & 1 & 0 & 4 & 419 \\
\hline 20 & 0 & 1 & 3 & 339 \\
\hline
\end{tabular}

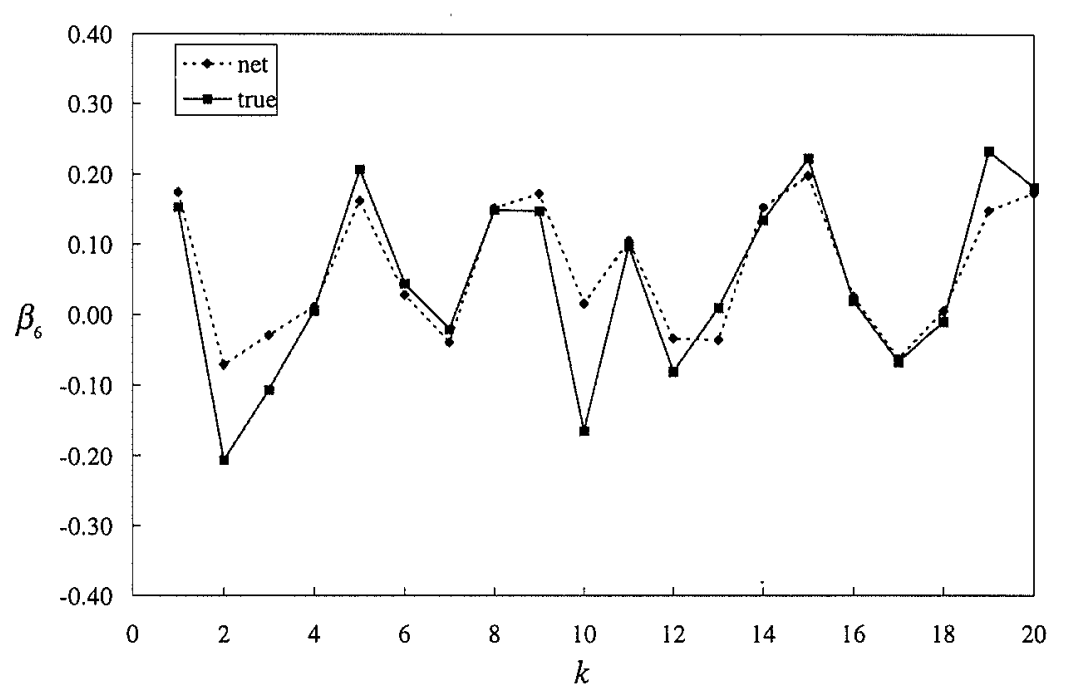

Fig. 8.

True values of $\beta_{6}$ (solid lines) and prediction by the $(5,4,1)$ feedback network (dotted lines). 


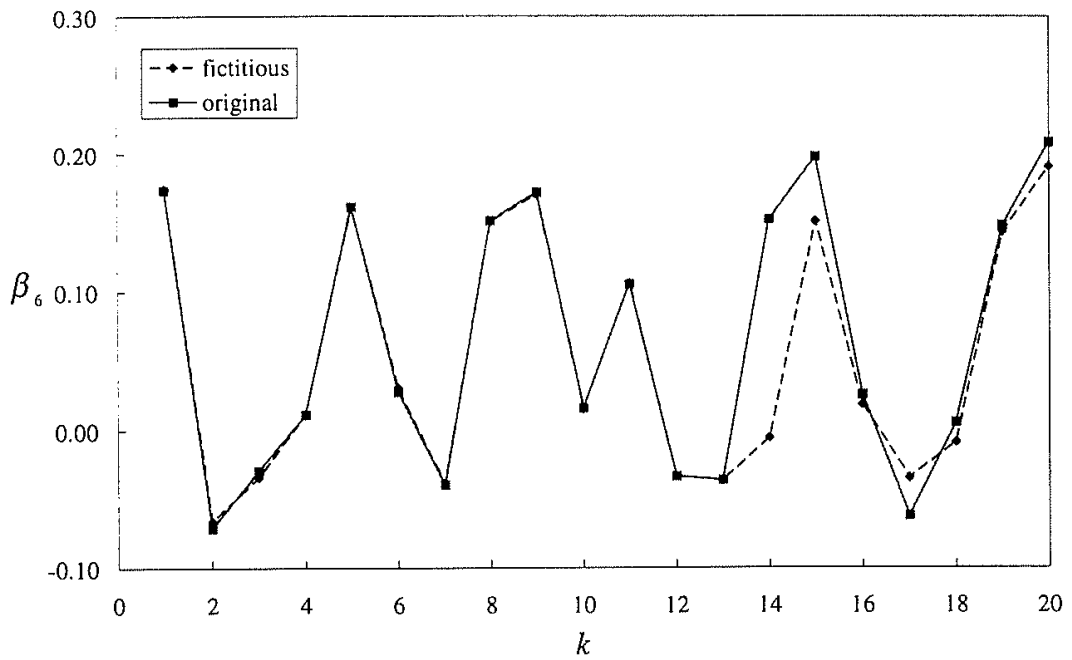

Fig. 9.

Predictions of the $(5,4,1)$ network for the original (solid line) and for a fictitious (dashed line) charging sequence.
Finally, an example on how the model can be used for off-line evaluation of charging programs is presented. The triggering effect of dumps of fine materials in the charging program is studied by means of a fictitious program, created by changing the 14 th dump in the sequence from fine to coarse coke. The $(5,4,1)$ network was evolved through two sequences of both the original and the fictitious program, and the results for the later sequence were compared (where the impact of the initial state has vanished). As depicted in Fig. 9, the change introduced has clearly affected the results: The model predicts that practically no cooling should be observed at $k=14$ for the new charging program, but the effect of the change vanishes quite soon.

\section{Discussion}

A model which aims at improved understanding of the burden distribution in the blast furnace has been presented. The idea behind the model is to utilize changes in the gas temperatures experienced at charging, measured by an above-burden probe, as an indication of the local layer thickness. The model has not yet focused on layer thickness estimation, but only on prediction of the (normalized) temperature drops in the center of the furnace. Given information about some central process variables, it has been demonstrated that the temperature drops can be predicted quite accurately. The models utilized in this study are neural networks, which have been trained on one data set and evaluated on another independent data set. Two models have been developed: An on-line feedforward model which requires recent measurements as inputs, and an off-line recurrent model with the predictions fed back as inputs. The latter model, which is autonomous, will be further developed for off-line evaluation of different charging programs and sequences.

The models presented are still preliminary since they are based on several gross simplifications. For instance, the effect of the movable armor position has not been considered, and the models do not yet predict the burden layer thickness. In the forthcoming work, the temperature drops at other points on the diagonal will be considered, as well as the influence of the radial distribution of the gas. ${ }^{23)} \mathrm{A}$ large amount of process data will be used to improve the generalization capabilities of the models.

\section{Acknowledgments}

The authors are grateful for the financial support from the SULA 2 project which is funded by the Technology Development Center of Finland (TEKES). Fundia Wire $\mathrm{Oy} \mathrm{Ab}$ is gratefully acknowledged for making available the data from the blast furnace.

\section{Nomenclature}

$a$ : parameter in discrete first order system, -

$A$ : heat transfer area, $\mathrm{m}^{2}$

$b$ : parameter in discrete first order system, ${ }^{\circ} \mathrm{C}$

$c$ : specific heat capacity, $\mathrm{kJ} /(\mathrm{kg} \mathrm{K})$

$C$ : heat capacity, $\mathrm{kJ} / \mathrm{K}$

$d:$ particle diameter, $\mathrm{m}$

$e$ : root mean square (rms) error, -

$f$ : function, -

$h$ : heat transfer coefficient, $\mathrm{kW} /\left(\mathrm{m}^{2} \mathrm{~K}\right)$

$k$ : dump number, -

$K$ : number of probe measurements, -

$l$ : sample index for hopper temperature, -

$m:$ mass, $\mathrm{kg}$

$M$ : number of observations, -

$n$ : number of observations in step response, -

$N$ : number of units in network model, -

$\mathrm{Nu}$ : Nusselt number, -

Pr: Prandtl number, -

$q^{-1}$ : backward shift operator, -

$Q:$ heat, $\mathrm{kJ}$

Re: Reynolds number, -

$t:$ time, min

$T$ : temperature, ${ }^{\circ} \mathrm{C}$

w: network weight vector, -

\section{Greek letters}

$\beta$ : normalized gas temperature change, -

$\gamma$ : binary variable for material, -

$\delta$ : binary variable for particle size, -

$\Delta:$ difference operator, -

$\varepsilon:$ porosity, -

$\lambda$ : thermal conductivity, $\mathrm{W} /(\mathrm{m} \mathrm{K})$

$\tau$ : time constant, min 
$\Psi:$ particle shape factor, -

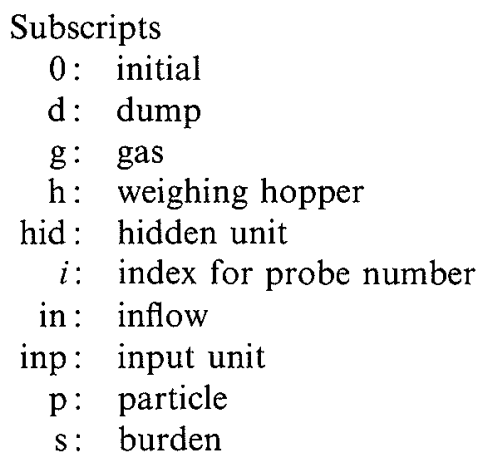

Other notations

A dot above a symbol denotes the time derivative (i.e. flow) and a hat $\left(^{-}\right)$an estimate.

\section{REFERENCES}

1) J. J. Poveromo and J. W. Hlinka: Proc. of 3rd Process Technology Conf. Pittsburgh, (1982), 166.

2) Blast Furnace Phenomena and Modeling, ed. by Y. Omori, Elsevier Applied Sciences, Essex, (1987).

3) K. Narita, S. Inaba, I. Kobayashi, K. Okimoto, M. Shimizu, T. Yabata and S. Tamada: Trans. Iron Steel Inst. Jpn., 19 (1979), 667.

4) K. Narita, S. Inaba, M. Shimizu, A Yamaguchi, I. Kobayashi and K. Okimoto: Trans. Iron Steel Inst. Jpn., 21 (1981), 405.

5) Y. Kajiwara, T. Inada, T. Tanaka and T. Jimbo: Sumitomo Search, 44 (1990), December, 249.

6) T. Inada, Y. Kajiwara and T. Tanaka: Proc. of 49th Ironmaking Conf., Detroit, (1990), 263.

7) M. Iizuka, S. Kajikawa, G. Nakatani, K. Wakimoto and K. Matsumura: Nippon Kokan Tech. Rep., (Overseas), 30(1980), 13.

8) R. Nicolle, C. Thirion, P. Pochopien and M. Le Scour: Proc. of 46th Ironmaking Conf., Pittsburgh, (1987), 217.

9) T. Iwamura, H. Sakimura, Y. Maki, T. Kawai and Y. Asano: Trans. Iron Steel Inst. Jpn., 22 (1982), 764.

10) H. J. Grisse: Steel Times Int., (1991), November, 612.

11) D. D. Mawhinney, A. Presser and T. G. Koselke: Proc. of 52nd Ironmaking Conf., Dallas, (1993), 563.

12) Metal Process Control (MPC): Steel Times Int., (1991), November, 611.

13) A. Poos, G. Danloy and R. Franssen: Proc. of 40th Ironmaking Conf., Toronto, (1981), 98.

14) G. Fleming, R. Schmit and P. Tonteling: Proc. of 42nd Ironmaking Conf., Atlanta, GA, (1983), 467.

15) J. Ohno, H. Yashiro, Y. Shirakawa, A. Tsuda, S. Watanabe, T. Hirata, M. Higuchi and M. Nakagome: IFAC Conf. on Automation in Mining, Mineral and Metal Processing, Tokyo, (1986), 353.

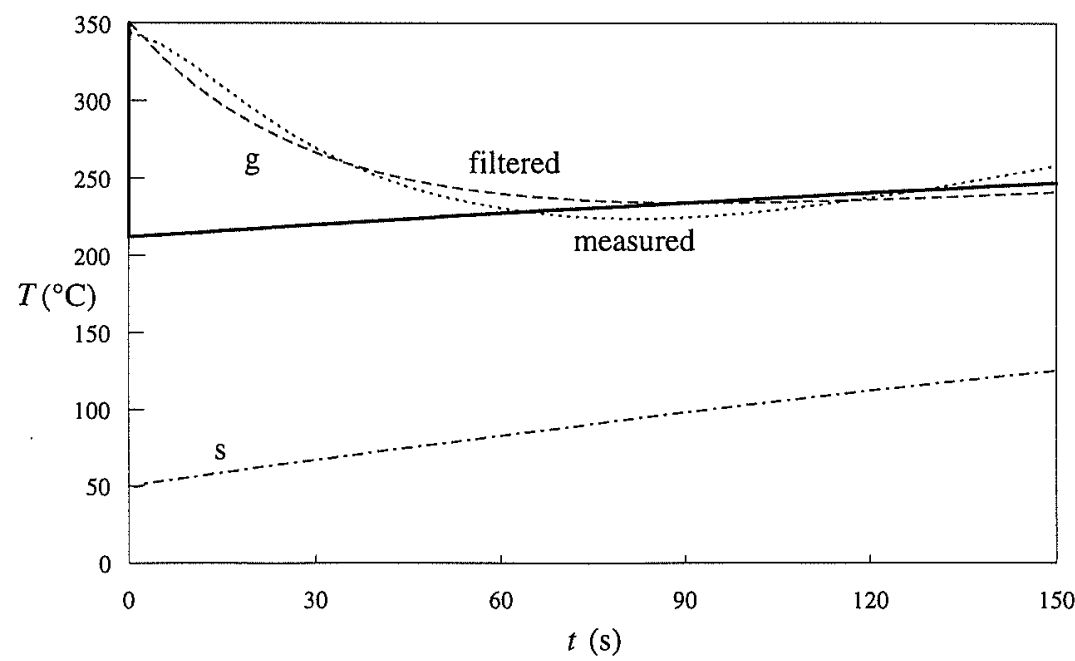

Fig. A1.

Temperature transients after a dump of coarse sinter at $t=0(\mathrm{~g}=$ gas, $\mathrm{s}=$ burden $)$. The solid line depicts the simulated gas temperature, and the dashed line the response after passing the signal through a first order filter with a time constant of $30 \mathrm{~s}$. The dotted line depicts a measured response in the blast furnace. 
trated. Using $C_{\mathrm{s}}=10 \mathrm{MJ} / \mathrm{kg}$ the time constant (in Eq. (6)) becomes $\tau=522 \mathrm{~s}$. The results are presented in Fig. A1. On the basis of short-term measurements from the probe (dotted line in the figure) it was found that there are some additional dynamics involved, since the recorded temperature drops are not immediate as in the simulations, but show a minimum about $80 \mathrm{~s}$ after the dump. The dynamics mainly originate from the measurement itself, because the thermocouple exchanges heat with the water-cooled probe by radiation. Moreover, some backmixing of the gas may occur before the probe, but this effect is believed to be rather small.

By processing the simulated temperature and making a comparison with the measured response, we may analyze whether the probe dynamics could be explained by, e.g., a first-order system. The dashed line in Fig. Al shows the response after passing $T_{\mathrm{g}}$ through a first order system with a time constant of $30 \mathrm{~s}$. The qualitative resemblance between this curve and the measurement (dotted line) indicates that the approach was successful. Also, it can be observed that for these (quite arbitrarily selected) conditions, the maximum change in the gas temperature appears between one and two minutes after the dump, which confirms the observation used in the modeling, (Sec. 2.1, Eq. (7)). However, it should be kept in mind that the formulae derived strictly hold only for the heat transfer immediately after the dump, when the gas entering the layer can be assumed to be characterized by a constant temperature. 